\title{
Novel Roles of Necroptosis Mediator Receptor-Interacting Protein Kinase 3 in Kidney Injury
}

\author{
Rie Uni ${ }^{\mathrm{a}}$ Mary E. Choi ${ }^{\mathrm{a}, \mathrm{b}}$ \\ aDivision of Nephrology and Hypertension, Joan and Sanford I. Weill Department of Medicine, Weill Cornell \\ Medicine, New York, NY, USA; ${ }^{b}$ Department of Medicine, New York-Presbyterian Weill Cornell Medical Center, New \\ York, NY, USA
}

\section{Keywords}

Acute kidney injury · Kidney fibrosis - Necroptosis .

Receptor-interacting protein kinase $3 \cdot$ Mitochondrial DNA

\section{Abstract}

Necroptosis is a programmed cell death that is characterized by regulated necrosis resulting in plasma membrane rupture and subsequent release of damage-associated molecular patterns (DAMPs). Receptor-interacting protein kinase 3 (RIPK3) is a key mediator of this pathway. Accumulating evidence supports a critical role of RIPK3 and the necroptosis pathway in various human diseases. In this review, we discuss recent investigations that have uncovered pathogenic roles of RIPK3 in both acute kidney injury (AKI) and kidney fibrosis. RIPK3 promotes kidney tubular injury via a mechanism involving mitochondrial dysfunction. Additionally, extracellular mitochondrial DNA, which is one of the necroptotic DAMPs, released from damaged mitochondria correlates with kidney tubular injury and represents a potential novel biomarker. RIPK3 also induces kidney fibrogenesis through AKT-dependent activation of the metabolic enzyme
ATP citrate lyase. Thus, the RIPK3-mediated necroptosis pathway may serve as a promising new therapeutic target in AKI and kidney fibrosis.

(c) 2021 S. Karger AG, Basel

\section{Introduction}

Acute kidney injury (AKI) is a common complication in critically ill patients that portends adverse outcomes as an episode of AKI is a risk factor for future development or progression of chronic kidney disease (CKD), ultimately leading to end-stage kidney disease [1]. To date, there are no specific pharmacological treatments for AKI and CKD. This represents a critical unmet medical need.

Contribution from the AKI and CRRT 2021 Symposium at the 26th International Conference on Advances in Critical Care Nephrology, A Virtual/Hybrid Event from San Diego, CA, USA, February 28-March 5, 2021. This symposium was supported in part by the NIDDK funded University of Alabama at Birmingham-University of California San Diego O'Brien Center for Acute Kidney Injury Research (P30DK079337).

Correspondence to:

Mary E.Choi, mechoi@med.cornell.edu 
Therefore, it is crucial to elucidate the mechanism of pathogenesis of AKI and CKD for developing new therapies. Here, we review recent findings of novel roles of the necroptosis mediator receptor-interacting protein kinase 3 (RIPK3) in AKI and kidney fibrosis.

\section{Necroptosis}

Necroptosis is a genetically regulated form of cell death that has emerged as an important pathway in many human diseases. In contrast to apoptosis, a well-documented programmed cell death characterized morphologically by DNA fragmentation and cell shrinkage, necroptosis is regulated necrosis with key features of cell swelling and plasma membrane rupture [2].

The necroptosis pathway is regulated by receptor-interacting protein kinase 1 (RIPK1) and RIPK3 and the downstream substrate mixed-lineage kinase domain-like (MLKL) pseudokinase. Although necroptosis and apoptosis are morphologically different, they are not completely independent. Tumor necrosis factor (TNF) receptor 1 (TNFR1) stimulation by TNF results in either caspase-8-dependent apoptosis or alternative activation of the necroptosis pathway depending on caspase- 8 activity (Fig. 1). Activated caspase-8 cleaves necroptosis mediators RIPK1 and RIPK3, while activating downstream caspase- 3 and -7 , and the cell undergoes apoptotic cell death. Inhibition of caspase- 8 prevents cleavage of the necroptosis mediators, thereby allowing RIPK1 and RIPK3 to interact with each other leading to auto- or transphosphorylation and assembly of the necrosome complex [2]. Ensuing RIPK3-mediated MLKL recruitment and phosphorylation promote MLKL oligomerization and translocation to the plasma membrane, inducing extracellular calcium influx and plasma membrane permeabilization $[3,4]$. Subsequently, necroptotic damage-associated molecular patterns (DAMPs) such as high-mobility group box 1, heat shock proteins, and mitochondrial DNA (mtDNA) are released. These extracellularly released DAMPs can activate infiltrating immune cells with subsequent cytokine release and promote inflammation. Although the role of RIPK3 is well-documented in canonical MLKL-dependent necroptosis pathway, recent investigations revealed that RIPK3 also contributes to various organ injury, including cardiac ischemia [5] and ventilator-induced lung injury [2] independent of MLKL. Accumulating evidence demonstrates MLKL-independent effects of RIPK3 in the pathogenesis of AKI via mitochondrial dysfunction and kidney fibrosis.

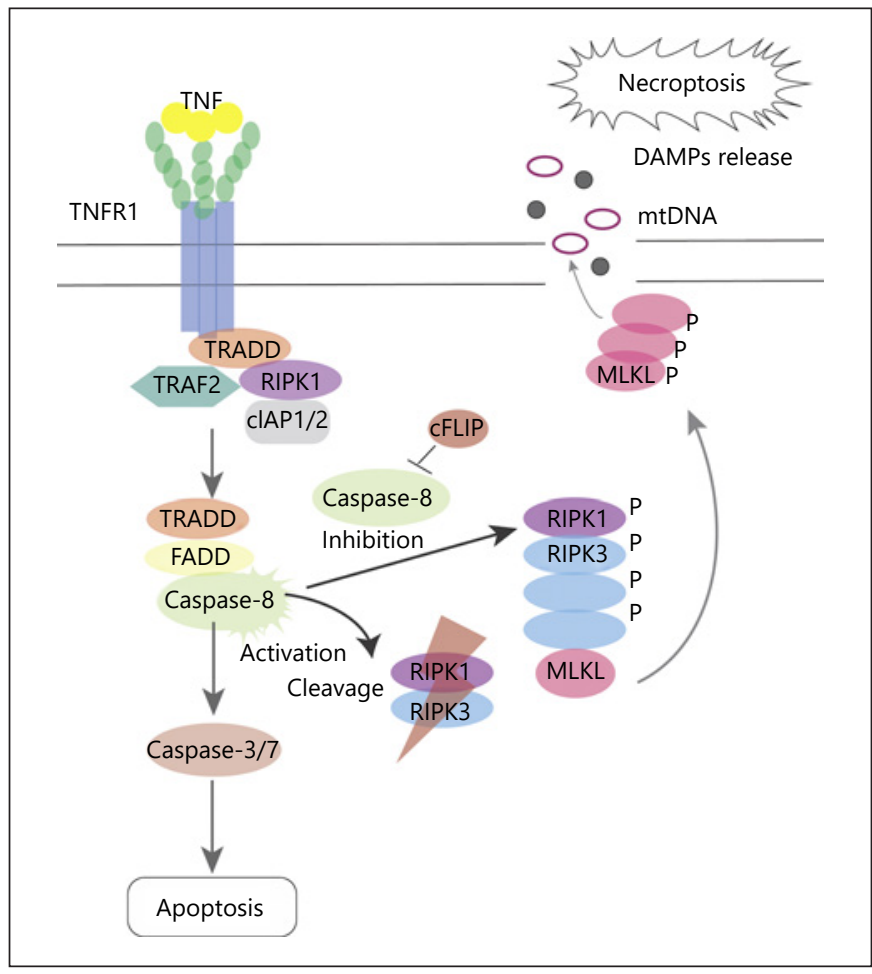

Fig. 1. Cross-talk between apoptosis and necroptosis pathways. The extrinsic apoptosis pathway is activated upon TNFR1 binding its ligand TNF and the recruitment of TRADD, TRAF2, cIAP1/2, and RIPK1. Subsequent recruitment of FADD and procaspase- 8 leads to caspase- 8 activation, which in turn activates downstream caspase-3/7 to induce apoptosis but cleaves RIPK1 and RIPK3 to block necroptosis. On the other hand, one of the antiapoptotic proteins cFLIP can heterodimerize with caspase-8, resulting in inhibition of caspase-8, enabling RIPK1 and RIPK3 auto- or transphosphorylation to form a necrosome complex and activate MLKL to induce necroptosis. Phosphorylated MLKL oligomerization and translocation to the plasma membrane lead to cell rupture and release of DAMPs including mtDNA. TNF, tumor necrosis factor; TNFR1, TNF receptor 1; TRADD, TNFR1-associated death domain; TRAF2, TNFR-associated factor 2; cIAP1/2, cellular inhibitor of apoptosis proteins 1 and 2; RIPK1, receptor-interacting protein kinase 1; RIPK3, receptor-interacting protein kinase 3; FADD, Fas-associated death domain; cFLIP, cellular FLICE-like inhibitory protein; MLKL, mixed-lineage kinase domain-like; DAMPs, damage-associated molecular patterns; mtDNA, mitochondrial DNA.

\section{RIPK3 in Sepsis-Induced AKI}

Sepsis is the leading cause of AKI in hospitalized critically ill patients, but the mechanism underlying the pathogenesis of sepsis-induced AKI is incompletely understood. Recent studies implicate the RIPK3-mediated necroptosis pathway in sepsis-associated organ injury. 
The loss of RIPK3 protected against lethal systemic inflammatory response syndrome and cecal ligation and puncture (CLP)-induced polymicrobial sepsis $[2,6]$. Utilizing the CLP model, we reported that sepsis induced RIPK3 expression in tubular epithelial cells, and RIPK3 but not MLKL knockdown protected against sepsis-induced AKI, as evidenced by lower kidney injury score with decreased kidney injury molecule- 1 expression and lower urinary lipocalin-2 and $\beta 2$-microglobulin levels in Ripk3 knockout ( $R i p k 3^{-1-}$ ) mice compared to wild-type $\left(R i p k 3^{+/+}\right)$mice after CLP [7]. Blood urea nitrogen levels were also significantly lower in Ripk $3^{-/-}$mice relative to Ripk $3^{+/+}$mice after CLP, and proteinuria was suppressed. Hence, Ripk3 deficiency protects the kidney against sepsis-associated tissue damage and kidney dysfunction [7]. Taken together, these findings indicate that RIPK3 promotes sepsis-induced AKI independently of the classical MLKL-dependent necroptosis pathway.

\section{RIPK3 and Mitochondrial Dysfunction}

Mitochondria are double membrane-bound organelles serving critical function in energy production by generating ATP. The importance of mitochondrial dysfunction in inflammatory response such as NLRP3 inflammasome activation and in the pathogenesis of sepsisinduced AKI has been described $[8,9]$. We demonstrated that RIPK3 acts upstream of mitochondrial dysfunction to induce oxidative stress and kidney injury in sepsis via upregulation of mitochondrial NADPH oxidase-4 (NOX4) through a post-transcriptional mechanism that involves intermolecular interactions between RIPK3 and NOX4 [7]. Loss of NOX4 attenuated kidney tubular injury similar to RIPK3 in the context of sepsis. NOX4 is a reactive oxygen species (ROS)-generating enzyme [10], and Ripk3 deficiency reduced ROS levels in septic mouse kidneys and in human proximal tubular epithelial cells after lipopolysaccharide challenge [7]. Ripk3 deficiency also mitigated sepsis-induced mitochondrial dysfunction both in vivo and in vitro. Thus, in sepsis-associated AKI, RIPK3 is induced, which in turn through NOX4 induces mitochondrial dysfunction and necroptosis.

mtDNA released from damaged mitochondria may be released from cells undergoing necroptosis, which is characterized by plasma membrane rupture. Extracellular mtDNA can serve as DAMPs to activate toll-like receptor 9 and induce inflammation. Cell-free mtDNA can be detected in biological fluids including plasma and urine and may also serve as a potential biomarker

Receptor-Interacting Protein Kinase 3 in Kidney Injury of mitochondrial injury. Elevated plasma mtDNA levels were reported in patients with critical illness, including sepsis and acute respiratory distress syndrome and found to be associated with mortality and improved risk prediction [11]. In our study, we demonstrated that both plasma and urinary mtDNA and RIPK3 levels were increased in patients with sepsis-induced AKI [7]. Additionally, RIPK3 expression in the kidney was also higher in both AKI with tubular injury and CKD with tubulointerstitial fibrosis in human kidney biopsy samples [12]. These results implicate that plasma and urinary RIPK3 and mtDNA could serve as potential biomarkers of kidney injury.

\section{Necroptosis Mediators in Kidney Fibrosis}

Kidney fibrosis is the hallmark of progressive CKD and a common pathogenic response to injury, irrespective of the etiology. The kidney parenchymal tissue is replaced with excessive extracellular matrix, leading to impaired kidney function and eventually end-stage kidney disease. Recent evidence supports the involvement of the necroptosis pathway in kidney fibrosis. Since RIPK1-deficient mice die within a few days after birth, investigations into the role of RIPK1 have employed the use of a specific inhibitor of RIPK1, necrostatin-1. Pharmacologic inhibition of necroptosis by administering necrostatin-1 attenuated kidney injury in the unilateral ureteral obstruction model of kidney fibrosis [13]. Utilizing genetic and pharmacologic inhibition of RIPK3 in 2 independent experimental murine models of kidney fibrosis induced by unilateral ureteral obstruction or adenine diet, we demonstrated that RIPK3mediated necroptosis pathway promoted kidney fibrosis [12]. Mice genetically deficient in Ripk3, but not $M l k l$, were protected against kidney fibrosis, suggesting that RIPK3 regulates fibrogenesis independently of MLKL. Others have also reported MLKL-independent functions of RIPK 3 in a murine model of inflammatory arthritis, wherein RIPK3 promoted interleukin-1 $\beta$ activation in an MLKL-independent fashion, indicating a potential role for RIPK3 in the regulation of inflammation [14]. In kidney fibrosis, we identified AKT as a downstream signaling target of RIPK3 and that RIPK3 promoted fibrogenesis through the AKT-dependent activation of the metabolic enzyme ATP citrate lyase (ACL) which catalyzes the synthesis of acetyl-CoA, an important metabolic substrate for fatty acid synthesis [12]. Moreover, inhibition of AKT or ACL suppressed 


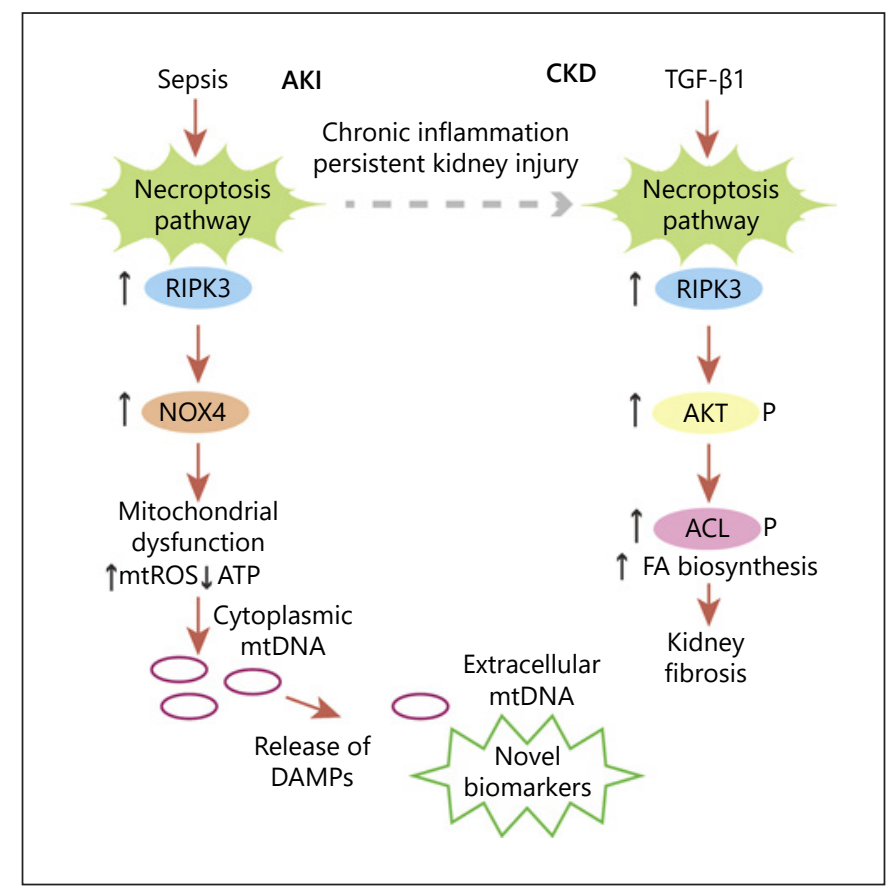

Fig. 2. Necroptosis mediator RIPK3 in $A K I$ and CKD. In septic AKI, RIPK3 promotes kidney tubular injury by inducing NOX4 and mitochondrial dysfunction with subsequently increased mtROS and reduced ATP production. Cytoplasmic mtDNA from damaged mitochondria can be released extracellularly as necroptotic DAMPs and may serve as novel biomarkers of kidney injury. In CKD, RIPK3 promotes kidney fibrosis via activation of AKT and ACL to modulate the fatty acid synthesis pathway. TGF- $\beta 1$, a potent pro-fibrotic cytokine, can also induce RIPK3 and activate the AKT/ACL pathway. RIPK3, receptor-interacting protein kinase 3; NOX4, NADPH oxidase-4; mtROS, mitochondrial reactive oxygen species; ATP, adenosine triphosphate; mtDNA, mitochondrial DNA; DAMPs, damage-associated molecular patterns; AKT, protein kinase B; ACL, ATP citrate lyase; FA, fatty acid; TGF- $\beta 1$, transforming growth factor-beta 1; AKI, acute kidney injury; CKD, chronic kidney disease.

TGF- $\beta 1$-dependent extracellular matrix production and myofibroblast differentiation, and pharmacologic inhibition of ACL suppressed kidney fibrosis, suggesting that drugs that inhibit ACL may have therapeutic benefit against kidney fibrosis. The TGF- $\beta 1$ signaling pathway is one of the most potent inducers of kidney fibrogenesis mediated via the canonical Smad signaling and the non-Smad signaling pathways, but TGF- $\beta 1$ is a multifunctional cytokine and may exert protective effects, for instance, via the induction of autophagy [15]. Our studies show that TGF- $\beta 1$ can also induce RIPK3 and activate the AKT/ACL pathway in a dose- and time-dependent fashion, while RIPK3 inhibition by
siRNA knockdown or chemical inhibitor blocked TGF$\beta 1$-dependent AKT/ACL phosphorylation and collagen stimulation [12]. Taken together, these findings suggest that RIPK3 plays a critical role in the pathogenesis of kidney fibrosis via alteration of fatty acid metabolism.

\section{Concluding Remarks and Future Directions}

The role of RIPK3 was originally described in the context of necroptosis, but emerging evidence supports the notion that RIPK3 acts beyond the classical MLKL-dependent necroptosis pathway. RIPK3 plays a critical role in the pathogenesis of both AKI and kidney fibrosis (Fig. 2). RIPK3 provokes kidney tubular injury by inducing NOX4 and mitochondrial dysfunction, leading to increased mitochondrial ROS and reduced ATP production in septic AKI. Damaged mitochondria release $\mathrm{mtD}$ $\mathrm{NA}$, and extracellular mtDNA may serve as a potential novel biomarker of kidney injury. RIPK3 also promotes kidney fibrosis via metabolic alteration independently of canonical MLKL-dependent necroptosis. RIPK3 via AKT/ACL activation and the fatty acid synthesis pathway induces kidney fibrosis, a hallmark of CKD. Thus, RIPK3 and mitochondrial-targeted approaches represent promising new therapeutic targets for treatment of AKI and CKD.

\section{Conflict of Interest Statement}

The spouse of M.E.C. is a cofounder and shareholder and serves on the Scientific Advisory Board of Proterris, Inc. R.U. has no conflicts of interest to disclose.

\section{Funding Sources}

M.E.C. is supported by the National Institute of Health (NIH) grants R01 HL133801, R01 HL132198, and R01 HL055330. R.U. receives fellowships from the Astellas Foundation for Research on Metabolic Disorders (Japan) and the Nakatomi Foundation (Japan).

\section{Author Contributions}

R.U. wrote the manuscript, and M.E.C. edited the manuscript. Both the authors revised and approved the final version of the manuscript. 


\section{References}

1 Chawla LS, Eggers PW, Star RA, Kimmel PL. Acute kidney injury and chronic kidney disease as interconnected syndromes. N Engl J Med. 2014 Jul;371(1):58-66.

2 Choi ME, Price DR, Ryter SW, Choi AMK. Necroptosis: a crucial pathogenic mediator of human disease. JCI Insight. 2019 Aug;4(15): e128834.

3 Dondelinger Y, Declercq W, Montessuit S, Roelandt R, Goncalves A, Bruggeman I, et al. MLKL compromises plasma membrane integrity by binding to phosphatidylinositol phosphates. Cell Rep. 2014 May;7(4):971-81.

4 Zhang Y, Chen X, Gueydan C, Han J. Plasma membrane changes during programmed cell deaths. Cell Res. 2018 Jan;28(1):9-21.

5 Zhang T, Zhang Y, Cui M, Jin L, Wang Y, Lv $\mathrm{F}$, et al. CaMKII is a RIP3 substrate mediating ischemia- and oxidative stress-induced myocardial necroptosis. Nat Med. 2016 Feb;22(2): 175-82.
6 Duprez L, Takahashi N, Van Hauwermeiren F, Vandendriessche B, Goossens V, Vanden Berghe T, et al. RIP kinase-dependent necrosis drives lethal systemic inflammatory response syndrome. Immunity. $2011 \mathrm{Dec} ; 35$ (6): 908-18.

7 Sureshbabu A, Patino E, Ma KC, Laursen K, Finkelsztein EJ, Akchurin O, et al. RIPK3 promotes sepsis-induced acute kidney injury via mitochondrial dysfunction. JCI Insight. 2018 Jun;3(11):e98411.

8 Sun J, Zhang J, Tian J, Virzì GM, Digvijay K, Cueto L, et al. Mitochondria in sepsis-induced AKI. J Am Soc Nephrol. 2019 Jul;30(7): 1151-61.

9 Moon JS, Nakahira K, Chung KP, DeNicola GM, Koo MJ, Pabón MA, et al. NOX4-dependent fatty acid oxidation promotes NLRP3 inflammasome activation in macrophages. Nat Med. 2016 Sep;22(9):1002-12.

10 Cadenas S. ROS and redox signaling in myocardial ischemia-reperfusion injury and cardioprotection. Free Radic Biol Med. 2018 Mar;117:76-89.
11 Nakahira K, Kyung SY, Rogers AJ, Gazourian L, Youn S, Massaro AF, et al. Circulating mitochondrial DNA in patients in the ICU as a marker of mortality: derivation and validation. PLoS Med. 2013 Dec;10(12):e1001577.

12 Imamura M, Moon J-S, Chung K-P, Nakahira K, Muthukumar T, Shingarev R, et al. RIPK3 promotes kidney fibrosis via AKT-dependent ATP citrate lyase. JCI Insight. 2018 Feb;3(3): e94979.

13 Xiao X, Du C, Yan Z, Shi Y, Duan H, Ren Y. Inhibition of necroptosis attenuates kidney inflammation and interstitial fibrosis induced by unilateral ureteral obstruction. Am J Nephrol. 2017;46(2):131-8.

14 Lawlor KE, Khan N, Mildenhall A, Gerlic M, Croker BA, D’Cruz AA, et al. RIPK3 promotes cell death and NLRP3 inflammasome activation in the absence of MLKL. Nat Commun. 2015 Feb;6:6282.

15 Sureshbabu A, Muhsin SA, Choi ME. TGF- $\beta$ signaling in the kidney: profibrotic and protective effects. Am J Physiol Renal Physiol. 2016 Apr;310(7):F596-F606. 